\title{
Black hole masses and accretion states in ULXs
}

\author{
Roberto Soria ${ }^{* \dagger}$ and Zdenka Kuncic ${ }^{\dagger}$ \\ ${ }^{*}$ MSSL, University College London, Holmbury St Mary, Dorking, Surrey, RH5 6NT, UK \\ ${ }^{\dagger}$ School of Physics, University of Sydney, NSW 2006, Australia
}

\begin{abstract}
We summarize indirect empirical arguments used for estimating black hole (BH) masses in ultraluminous X-ray sources (ULXs). The interpretation of the X-ray data is still too modeldependent to provide tight constraints, but masses $\lesssim 100 M_{\odot}$ seem the most likely. It is getting clearer that ULXs do not show the same evolutionary sequence between canonical spectral states as stellar-mass BHs, nor the same timescale for state transitions. Most ULX spectra are consistent either with a power-law-dominated state (apparently identical to the canonical low/hard state), or with a very high state (or slim-disk state). Despite often showing luminosity variability, there is little evidence of ULXs settling into a canonical high/soft state, dominated by a standard disk (diskblackbody spectrum). It is possible that the mass accretion rate (but not necessarily the luminosity) is always larger than Eddington; but there may be additional physical differences between stellarmass BHs and ULXs, which disfavour transitions to the standard-disk, radio-quiet state in the latter class. We speculate that the hard state in ULXs is associated with jet or magnetic processes rather than an ADAF, can persist up to accretion rates $\approx$ Eddington, and can lead directly to the very high state.
\end{abstract}

Keywords: Black holes - X-ray binaries - Infall, accretion, and accretion disks - X-ray sources PACS: 97.60.Lf, 97.80.Jp, 98.62.Mw, 98.70.Qy

\section{INDIRECT BLACK HOLE MASS ESTIMATES}

No kinematic (optical spectroscopic) mass measurements exist yet for black holes (BHs) in ultraluminous X-ray sources (ULXs), although a program of phase-resolved VLT observations for NGC $1313 \mathrm{X}-2$ is currently under way (Grisé et al., in preparation). Indirect, model-dependent methods for estimating $\mathrm{BH}$ masses include:

1. assuming that the absorption-corrected isotropic $\mathrm{X}$-ray luminosity does not exceed the isotropic Eddington luminosity of the $\mathrm{BH}: 4 \pi d^{2} f_{\mathrm{X}} \lesssim L_{\mathrm{Edd}} \approx$ $1.3 \times 10^{38}\left(M / M_{\odot}\right)$ erg $\mathrm{s}^{-1}$. The estimated mass $M$ is only an upper limit if we allow for moderate super-Eddington luminosity or unisotropic emission (which is the case even for a standard accretion disk without beaming). Hence, a factor $\sim 1 / 2-1 / 4$ is sometimes implicitly allowed for, in the mass estimate;

2. using a standard disk model to relate the peak temperature of a thermal spectral component to the innermost stable circular orbit of the $\mathrm{BH}$, and to the $\mathrm{BH}$ mass [1];

3. comparing caracteristic variability timescales in ULXs with those in Galactic BHs and AGN, using the empirical scaling $\tau \sim M$;

4. associating ULX spectral states with the "canonical" BH state classification [2]; the assumption here is that transitions between states occur at fixed values or ranges of the self-similar accretion parameter $\dot{m} \equiv \dot{M} / \dot{M}_{\text {Edd }} \approx\left(0.1 c^{2} \dot{M}\right) / L_{\text {Edd }}$. Hence, $\dot{m} \sim(0.1 / \eta)\left(L_{\mathrm{X}} / M\right)$, using $L_{\mathrm{X}}$ as a proxy for the accretion rate. 
Argument 1) suggests an upper limit of $\approx 100 M_{\odot}$ to the BH mass in ULXs, except perhaps for a handful of sources [3] with $L_{X} \approx 0.5-1.5 \times 10^{41}$, which may require intermediate-mass BHs or substantial beaming. A ULX in NGC 1365 is a good example of a source that may reach its Eddington limit at $L_{X} \approx 3 \times 10^{40} \mathrm{erg} \mathrm{s}^{-1}$, triggering an outflow that blows away the accreting matter [4]. Such an upper mass limit is consistent with the maximum $\mathrm{BH}$ mass $\left(M \approx 70 M_{\odot}\right)$ expected from individual stellar processes, i.e. direct collapse of a metal-poor, massive star [5].

\section{SOFT AND HARD STATES}

The other three arguments depend, more or less critically, on whether ULX spectral states follow the canonical classification for stellar-mass BHs. In this scenario, accreting BHs cycle between a low/hard state (power-law dominated, with photon index $\Gamma \approx 1.5-$ 2 ), a high/soft state (disk dominated, with an additional weak, steep power law with $\Gamma \approx$ 2.5-3.5) and, occasionally, a very high state. The high/soft state is where Galactic BHs spend the majority of their time during their luminous phases $\left(0.1 L_{\text {Edd }} \lesssim L_{\mathrm{X}} \lesssim L_{\text {Edd }}\right)$.

Conversely, most ULXs are found in a state dominated by a broad, "power-law-like" component, with photon indices $1.5 \lesssim \Gamma \lesssim 3$; the index distribution peaks at $\Gamma \approx 1.7-2$ $[6,7]$. There is no gap or dichotomy between the flat and steep power-law sources. One or two additional features are sometimes present [8]: i) a soft excess (probably direct thermal emission from a standard disk), usually at low temperatures $\left(0.1 \lesssim k T_{\text {in }} \lesssim 0.2\right.$ $\mathrm{keV}$ ), which contributes only 10-20 per cent of the X-ray luminosity; ii) a downward curvature or steepening of the spectrum at energies $\gtrsim 5 \mathrm{keV}$. The presence or absence of those two components in a given source often depends on the signal-to-noise of the observation (hence, soft thermal components are more likely to be found in the brightest sources), and the amount of column density $N_{\mathrm{H}}$ which can mask the soft thermal component. No other properties (such as spatial distribution or optical counterpart) have been found so far that can separate ULXs with a soft X-ray excess and/or spectral curvature from those in which those features are not detected.

Examples of ULXs in a classical high/soft state, dominated by a standard diskblackbody spectrum, are very rare: only $\sim 10$ per cent of the sources with $L_{X}>10^{39} \mathrm{erg}$ $\mathrm{s}^{-1}$ in [6]. The best-studied cases include M 81 X-6 [9], and two sources in the colliding galaxies NGC 4485/4490 [10]. In all three cases, the fitted disk-blackbody temperatures are $k T_{\text {in }} \approx 1-1.5 \mathrm{keV}$, for an emitted luminosity $L_{\mathrm{X}} \lesssim 4 \times 10^{39} \mathrm{erg} \mathrm{s}^{-1}$. Thus, those sources can easily be classified as the extreme end of the stellar-mass BH distribution. In other studies [7], some ULXs have been assigned to the high/soft state because a diskblackbody component was detected in their X-ray spectra: but crucially, in all those cases, the thermal component was relatively unimportant with respect to the powerlaw component, unlike the classical high/soft state of Galactic BHs. Another group of ULXs, including many of those with $L_{X} \approx 10^{40} \mathrm{erg} \mathrm{s}^{-1}$ (e.g., IC 342 X-1 [11], and a few more discussed in [8]), can be fitted equally well with a dominant disk-blackbody or similar thermal components, with $k T_{\text {in }} \approx 1.5-2.5 \mathrm{keV}$, or with a broken power-law or exponential cutoff above $\approx 5-7 \mathrm{keV}$. Again, this group of sources cannot be included in the classical high/soft state: their temperatures suggest that if their dominant X-ray emission component comes from the accretion disk, it must be heavily Comptonized, 
or in any case substantially different from the standard Shakura-Sunyaev spectrum [12]. In the classical state classification, they belong more properly to the very high state (or perhaps the slim-disk state; see the next section) [13]. Finally, a handful of "super-soft" ULXs, (e.g., one in M 101 [14] and another in NGC 4631 [15]), have a thermal spectrum with $k T \approx 70 \mathrm{eV}$; there have been suggestions that they represent intermediate-mass BHs in the canonical high/soft state, but the most likely scenario is a transient, superEddington, nova-like source (a transient massive outflow from a white dwarf or from the accretion disk of a stellar-mass object).

Not only is it difficult to identify well-defined, canonical low/hard and high/soft states in the ULX population on a statistical basis, but it is also difficult to pinpoint transitions between canonical states in individual sources (unlike the case of stellar-mass BHs). For example, NGC $1365 \mathrm{X}-1$ has been studied at various luminosity levels, varying between $L_{\mathrm{X}} \approx 3 \times 10^{39} \mathrm{erg} \mathrm{s}^{-1}$ and $L_{\mathrm{X}} \approx 3 \times 10^{40} \mathrm{erg} \mathrm{s}^{-1}$, but its spectrum is always dominated by a power-law with $\Gamma \approx 1.5-1.9$, with an additional (non-dominant) thermal component on some occasions [4]. Clearly, this system is never in the high/soft state. But assigning its behaviour either to the very high state or the low/hard state is also problematic, given the large range of luminosities.

In summary, there is a clear lack of ULXs in a high/soft state (disk-blackbody component contributing $>50$ per cent of the X-ray luminosity) with $3 \times 10^{39} \lesssim L_{X} \lesssim 2 \times 10^{40}$ erg s$~^{-1}$ and $0.3 \lesssim k T_{\text {in }} \lesssim 0.9 \mathrm{keV}$ (see also the luminosity-temperature plots in [16] and [17]). That is the region of the parameter space where we would find accreting BHs with masses $\approx 30-100 M_{\odot}$ in their high/soft state, using the same standard-disk scaling and evolutionary track $\left(L_{\text {disk }} \sim T_{\text {in }}^{4}\right)$ that applies to Galactic BHs in that state.

Those findings suggest that either BHs in that mass range do not exist (in contradiction with the Eddington-luminosity argument outlined earlier), or they do not spend much time in the standard-disk dominated state. In the former scenario, the ULX population would consist either of beamed stellar-mass BHs, or of intermediate-mass BHs $\left(M \sim 1000 M_{\odot}\right)$. But both these possibilities are problematic. Strong beaming seems inconsistent with the quasi-isotropic powering of ionized nebulae around some ULXs [18, 19]; it is also inconsistent with quasi-periodic oscillations found in some ULXs, for example NGC 5408 X-1 [20]. Intermediate-mass BHs require untested formation mechanisms, and are difficult to reconcile with the luminosity and spatial distribution of ULXs (more consistent with the upper end of high-mass X-ray binaries).

Therefore, we suggest that the lack of ULXs in that range of temperatures and luminosities is more likely due to the fact that they do not settle in a canonical high/soft state, and their X-ray spectral appearance is always heavily modified by some form of inverse Compton scattering. In that case, the argument is then whether most ULXs belong to the low/hard state or the very high state (as we mentioned earlier, there is no evidence for separate softer and harder populations, for example a different spatial distribution or a gap in luminosities). The occasional presence of a faint, and sometimes low-temperature thermal component can be consistent with both states: in the low/hard state, the standard disk may be truncated at radii much larger than the innermost stable orbit; but a cooler, fainter disk component is also found in the very high state of Galactic BHs (the best example being XTE J1550-564, [21, 22]). The Comptonizing corona is much hotter in the low/hard state, producing unbroken power-law spectra up to $\sim 100$ $\mathrm{keV}$; therefore, ULXs with a spectral curvature or break at $\sim 5-10 \mathrm{keV}$ are more likely 
to be in the very high state, if we want to apply the canonical scheme. The main reason why the low/hard and the very high state are easy to distinguish in Galactic sources is because the former occurs only at $L_{\mathrm{X}} \lesssim$ a few per cent of $L_{\mathrm{Edd}}$ (when the accretion rate is thought to be too low to permit a disk-dominated state), and the latter at $L_{X} \sim L_{\text {Edd }}$ (when the accretion rate is too high for a standard disk). If this were the case for ULXs, we would be forced to conclude that the sources in the very high state have masses $\lesssim 100 M_{\odot}$ and those in the low/hard state have masses $>1000 M_{\odot}$, as suggested in [7]. Instead, we speculate that in ULXs, in the absence of a canonical high/soft state, the low/hard state and the very high state can be contiguous, or perhaps even that there is no fundamental qualitative difference betwen the two, so that sources can appear to have a "low/hard" spectrum even for luminosities approaching the Eddington limit.

\section{WHERE IS THE ACCRETION DISK?}

There is no lack of physical and phenomenological models to explain why the Xray spectrum cannot be dominated by a standard disk at accretion rates $\dot{m} \gtrsim 1$. The presence of non-dominant, relatively cool disk-blackbody emission in addition to a stronger, broader power-law-like component can be interpreted qualitatively as a twophase structure: a standard disk directly visible outside a transition radius $R_{\mathrm{c}}$, and a "modified" (e.g., heavily Comptonized) disk or flow at $R<R_{\mathrm{c}}$. Typically, the broader, dominant spectral component accounts for $\sim 90$ per cent of the radiated power, hence we expect $R_{\mathrm{c}} \sim 50-100 R_{\mathrm{g}} \sim 10 R_{\mathrm{ISCO}}$. The fitted temperature of the soft component (outer disk) is then expected to be $k T_{\text {in }} \approx k T\left(R_{\mathrm{c}}\right) \approx k T\left(R_{\mathrm{ISCO}}\right)\left(R / R_{\mathrm{ISCO}}\right)^{-3 / 4} \sim 0.1-0.3 \mathrm{keV}$ for $M \sim 10-100 M_{\odot}$. (If $L_{\mathrm{X}} \approx 10^{40} \mathrm{erg} \mathrm{s}^{-1}$, masses nearer the upper limit of this range are also more consistent with the Eddington-luminosity argument).

This simple argument can explain the characteristic temperature and luminosity of the soft excess found in many ULXs without invoking intermediate-mass BHs. More importantly, there is evidence that this is what happened in the Galactic BH XTE J1550-564 $\left(M \approx 10 M_{\odot}\right)$ when it reached the very high state in the 1998 outburst [21, 23, 24, 22]. In that phase of the outburst, the spectrum became dominated by a power-law-like component; the fitted peak temperature of the disk-blackbody component decreased from $\approx 1$ $\mathrm{keV}$ (as expected for a $\mathrm{BH}$ of this mass) to $\approx 0.4 \mathrm{keV}$, but without a decrease in the fitted disk-blackbody luminosity1. This finding can be explained if the disk-blackbody component has an increasing (receding) inner radius $R_{\mathrm{c}}$ at increasing accretion rate $\dot{m}$. Crucially, during that same days, a low-frequency QPO was detected [26], with a varying frequency, inversely correlated with the fitted inner-disk radius [22]. This is consistent with the QPO frequency being related to the transition radius $R_{\mathrm{c}}$ between the outer standard disk and the inner Comptonizing region, and provides further evidence in support

\footnotetext{
1 The reliability of the fitted disk-blackbody temperatures in the RXTE/PCA data from the 1998 outburst, originally presented in [25], has sometimes been questioned or dismissed: e.g., J. McClintock, priv. comm.; T. Belloni, priv. comm. On the other hand, independent re-analysis of the data by C. Done and collaborators has confirmed the low temperature values. In our opinion, the simultaneous correlated increase in the observed QPO frequency over the same days provides convincing evidence that both sets of measurements are reliable and are tracing a real physical effect.
} 
of a receding $R_{\mathrm{c}}$ at high accretion rates. There are various scenarios or physical models that can explain a transition between standard disk and non-standard inflow, with a transition radus moving outwards at increasing $\dot{m}$. Some of them are:

1. the inner disk is covered or partly replaced by a warm corona with $k T \sim$ a few $\mathrm{keV}$ (much cooler than in the low/hard state, leading to a noticeable break at energies $\gtrsim 5 \mathrm{keV}$ ), and scattering optical depth $\tau^{\text {es }} \sim$ a few [24, 27, 8, 28]. $R_{\mathrm{c}}$ is interpreted as the outer boundary of the corona, beyond which the geometrically-thin standard disk is visible. This scenario requires that most accretion power be dissipated in the corona rather than the inner disk, or be transferred efficiently to the corona rather than directly radiated. A related version of this scenario was proposed by [29], with the inner disk covered by a hotter $(k T \sim 100 \mathrm{keV})$, moderately optically-thin $\left(\tau^{\text {es }} \lesssim 1\right)$ corona, and high albedo at the photoionized inner-disk surface; this, and the dissipation of most accretion power inside the corona, ensures a non-thermal outgoing X-ray spectrum from the inner region. This model is more suitable for ULX spectra with a soft excess but no high-energy breaks; for example those that may have been classified in the low/hard state, if we used canonical states.

2. a physical model related to the previous coronal scenarios is based on the standard disk itself becoming hotter, effectively thin $\left(\tau_{v}^{\text {eff }} \approx \sqrt{\tau_{v}^{\text {ff }} \tau^{\text {es }}} \lesssim 1\right)$ but still thick to scattering $\left(\tau^{\mathrm{es}} \sim 10\right)$ in the inner region, when $\dot{m} \approx 1$ [12, 30, 31, 32, 33, 34]. It was already known since [12] that in the radiation-pressure-dominated zone of the disk, the electron density $n_{e} \sim \dot{m}^{-2}$; this is why the inner part of the disk eventually becomes effectively thin at high accretion rates. When that happens, the inner disk radiates less efficiently than a blackbody at a given temperature, so this is compensated by an increase of the temperature, up to $\sim$ a few $\mathrm{keV}$. The outgoing spectrum becomes heavily Comptonized, with a power-law-like appearance in the 2-10 keV band. Unlike the corona models, here there is no need to invoke a separate physical object covering or replacing the disk: it is the inner disk itself that morphs into a geometrically-thick, scattering-dominated region, with physical parameters consistent with those required for ULX spectral fits. In our phenomenological application to ULXs, $R_{\mathrm{c}}$ can be identified with the thick/thin transition radius. A self-similar analytic approximation from [12] suggests $R_{\mathrm{c}} / R_{\mathrm{ISCO}} \approx 12 \alpha^{34 / 93} m^{2 / 93} \dot{m}^{32 / 39}\left[1-\left(R_{\mathrm{c}} / R_{\mathrm{ISCO}}\right)^{-1 / 2}\right]^{64 / 93} \sim$ a few $\dot{m}^{0.69}$, where $m$ is the BH mass in solar units. Hence, the transition between standard disk and Comptonizing region appears when the accretion rate approaches Eddington; moves outwards for increasing $\dot{m}$ (in agreement with the very-high-state interpretation of XTE J1550-564 and some ULXs); and the effect is stronger for a high viscosity parameter $\alpha$;

3 . at super-Eddington accretion rates $(\dot{m} \gtrsim 2)$, the inner region of the accretion flow becomes a "slim disk"[36]: an optically-thick solution with energy advection and $H / R \sim 1$. Characteristic slim-disk temperatures are $k T_{\text {in }} \approx 1.5-2.5 \mathrm{keV}$, with

\footnotetext{
2 The numerical coefficient in front of this expression differs from the one in [12] because we are using a higher free-free absorption coefficient, suitable to cosmic abundances; see also [35].
} 
$L_{\text {disk }} \sim T_{\text {in }}^{2}$; the radial temperature profile in the disk is flatter than $R^{-3 / 4}$. The emitted luminosity saturates at $\sim$ a few $L_{\text {Edd }}$ for $\dot{m} \sim 10$, partly because of photon trapping; the transition between outer standard disk and photon-trapping region occurs at $R_{\mathrm{c}} \sim(\dot{m})^{2} R_{\mathrm{g}}$ [37, 38]. In this scenario, the slim disk provides the dominant broad component with an exponential cutoff above $\sim 5 \mathrm{keV}$. It predicts similar spectra to those expected from Comptonization in a warm, thick corona [39, 13]. Hence, it is more suitable for ULXs that show strong spectral curvature (which can also be modelled with a dominant disk-blackbody component at $k T_{\text {in }} \approx 1.5-2.5 \mathrm{keV}$, e.g., [8]); for example, IC 342 X-1 [11, 40]. In the slim-disk scenarios, BH masses in ULXs are $\sim 30-100 M_{\odot}$. When the spectral curvature is less prominent, or occurs $>5 \mathrm{keV}$, Comptonization models may be more suitable [13];

4. in addition to the thick/thin transition discussed earlier, standard disks have another characteristic radius that becomes relevant for $\dot{m} \gtrsim 1$ : the spherization radius $R_{\mathrm{sp}} \sim(9 / 4) \dot{m} R_{\mathrm{ISCO}}$. At $R \approx R_{\mathrm{sp}}$, the thin-disk approximation breaks down $(H / R \gtrsim 0.5)$ because radiation pressure dominates over gravity; strong outflows are launched at $R \lesssim R_{\mathrm{sp}}$. The total disk luminosity is $\approx L_{\mathrm{Edd}} \times(1+\ln \dot{m})$. Of this, $L \approx L_{\mathrm{Edd}}$ is released at $R>R_{\mathrm{sp}}$ and $L \approx(\ln \dot{m}) L_{\mathrm{Edd}}$ in the outflow region; the latter component can also be mildly beamed by the outflow itself, if seen face-on. It was suggested [41, 42] that the spherization radius corresponds to the characteristic transition radius $R_{\mathrm{c}}$ between outer (standard) disk and inner inflow, required for most ULX models. For accretion rates $\dot{m} \sim 10-100$ and stellar-mass BHs, the model reproduces the characteristic temperature of the soft excess $\left(k T\left(R_{\mathrm{sp}}\right)\right)$, the total luminosity and the relative contribution of the inner and outer regions. However, it may have more difficulties in explaining the power-law-like (or cutoff power-law) spectrum of the emission from the inner region. Also, the low intrinsic $N_{\mathrm{H}}$ fitted to most ULX spectra suggests that they are not seen through strong outflows;

5. finally, the centrifugal boundary layer model [43] may be a promising (and so far, not fully exploited) tool to understand some ULX spectra. The boundary layer is caused by an adjustment of the Keplerian disk to the sub-Keplerian boundary conditions near the $\mathrm{BH}$; its position depends on $\dot{m}$ and viscosity, through the Reynolds number [44]. The boundary layer consists of standing or oscillating shock waves that accelerate electrons very efficiently, producing a non-thermal (powerlaw) spectrum [45], in addition to the seed disk-blackbody component from the standard outer disk, before the shock. This model predicts testable correlations between QPO frequencies (oscillations of the bounday layer) and photon index of the power-law component.

\section{CONCLUSIONS}

X-ray spectral and timing studies have not yet provided a tight constraint on ULX BH masses, because they are still too model-dependent. The simplest Eddington-limit argument suggests $M \lesssim 100 M_{\odot}$ for all but a handful of ULXs; it remains the least controversial, and is also in agreement with most spectral models.

The most significant finding from X-ray spectroscopy is that ULXs are much less likely than Galactic BHs to settle in the canonical high/soft state, dominated by a 
standard disk. Some ULX spectra are dominated by a hard power-law (apparently identical to canonical low/hard state spectra); others have a softer (steeper) spectrum, sometimes with a soft thermal excess and a high-energy downturn, consistent with heavily Comptonized emission from a standard disk, or a slim disk.

There are various plausible models that may explain the luminosity and spectral shape for accretion rates $\dot{m} \gtrsim 1$. However, many ULXs show flux variability by at least a factor of a few; some are transients, so we know that the accretion rate is sometimes strongly reduced or switched off. We would expect to find some of them, at some epochs, in a canonical high/soft state, when $\dot{m} \sim 0.1-1$. This would also give us a chance to identify their standard $L_{\text {disk }} \sim T_{\text {in }}^{4}$ track and better constrain their mass. But this is not the case, although some ULXs have been seen to switch between a hard power-law state and a steeper spectrum with high-energy curvature. Furthermore, ULXs with a pure hard power-law spectrum are not significantly less luminous than those with a softer spectrum or a soft excess; some of them also exceed $10^{40} \mathrm{erg} \mathrm{s}^{-1}$. This is unlike the canonical low/hard state in Galactic BHs.

We speculate that there must be a fundamental physical difference between Galactic BHs and ULXs, which prevents the latter from settling into a long-duration diskdominated state. For example, ULXs may switch between harder and softer states depending on the optical depth and temperature of the Comptonizing region but would always be dominated by Comptonized emission. If ULX BHs have masses $\sim 30-100 M_{\odot}$, it may seem unlikely that such a small mass difference may suppress the disk-dominated state. After all, standard disks are seen in AGN with masses $\sim 10^{6}-10^{9} M_{\odot}$. The type of donor star (probably OB stars in ULXs) may have an effect on the duty cycle, keeping them in a bright state for longer periods of time than soft X-ray transients (powered by low-mass giants). But it is not clear why it would have an effect on the disk stability.

Perhaps the key is in the nature of the low/hard state. If/when it consists of a truncated disk replaced by a radiatively-inefficient, advection-dominated flow, it has to be limited to accretion rates $\dot{m} \lesssim 0.01$. But it is likely that in some Galactic BHs, the low/hard state has a fully-formed disk with a jet [46]. If so, the key element that defines the low/hard state is that most of the accretion power is carried out non-radiatively, in a jet, wind or Poynting flux [47]. The transition from the low/hard to the high/soft state would correspond to the suppression of the jet or Poynting flux, with ejections or flaring resuming as the source enters the very high state. (We do not know whether ULXs also possess a radio-quiet state, or it is suppressed together with the high/soft state). Therefore, we speculate that ULXs can remain in a power-law dominated state (similar to the low/hard state) up to $\dot{m} \sim 1$ and then switch directly to a very high state or outflowdominated or slim-disk state, as the accretion rate (but not necessarily the luminosity) increases above Eddington.

\section{REFERENCES}

1. K. Makishima, ApJ 535, 632-643 (2000).

2. J. E. McClintock and R. A. Remillard, "Black hole binaries," in Compact stellar X-ray sources, edited by W. Lewin \& M. van der Klis, Cambridge Astrophysics Series, No. 39, Cambridge University Press, Cambridge, 2006, pp.157-213.

3. A. R. King and W. Dehnen, MNRAS 357, 275-278 (2005). 
4. R. Soria, A. Baldi, G. Risaliti, G. Fabbiano, A. R. King, V. La Parola and A. Zezas, MNRAS 379, 1313-1324 (2007).

5. L. R. Yungelson, E. P. J. van den Heuvel, J. S. Vink, S. F. Portegies Zwart and A. de Koter, A\&A 477, 223-237 (2008).

6. D. A. Swartz, K. K. Ghosh, A. F. Tennant and K. Wu, ApJS 154, 519-539 (2004).

7. L. M. Winter, R. F. Mushotzky and C. S. Reynolds, ApJ 649, 730-752 (2006).

8. A.-M. Stobbart, T. P. Roberts and J. Wilms, MNRAS 368, 397-413 (2006).

9. D. A. Swartz, K. K. Ghosh, M. L. McCollough, T. G. Pannuti, A. F. Tennant and K. Wu, ApJS 144, 213-242 (2003),

10. T. P. Roberts, R. S. Warwick, J. M. Ward and S. S. Murray, MNRAS 337, 677-692 (2002).

11. A. Kubota, C. Done and K. Makishima, MNRAS 337, L11-L15 (2002).

12. N. I. Shakura and R. A. Sunyaev, $A \& A$ 24, 337-355 (1973).

13. K. Makishima, PThPS 169, 214-220 (2007).

14. A. K. H. Kong, R. Di Stefano and F. Yuan, ApJ 617, L49-L52 (2004).

15. S. Carpano, A. M. T. Pollock, A. R. King, J. Wilms and M. Ehle, A\&A 471, L55-L58 (2007).

16. J. M. Miller, A. C. Fabian and M. C. Miller, ApJ 614, L117-L120 (2004).

17. H. Feng and P. Kaaret, ApJ 633, 1052-1063 (2005).

18. P. Kaaret, M. J. Ward and A. Zezas, MNRAS 351, L83-L88 (2004).

19. M. W. Pakull and F. Grisé, in A Population Explosion: the Nature \& Evolution of X-ray Binaries in Diverse Environments, AIP Conference Proceedings, Volume 1010, pp. 303-307 (2008).

20. T. E. Strohmayer, R. F. Mushotzky, L. Winter, R. Soria, P. Uttley and M. Cropper, ApJ 660, 580-586 (2007).

21. A. Kubota and C. Done, MNRAS 353, 980-990 (2004).

22. R. Soria, $A p \& S S$ 311, 213-222 (2007).

23. A. Kubota and K. Makishima, ApJ 601, 428-438 (2004).

24. C. Done and A. Kubota, MNRAS 371, 1216-1230 (2006).

25. G. J. Sobczak, J. E. McClintock, R. A. Remillard, W. Cui, A. M. Levine, E. H. Morgan, J. A. Orosz and C. D. Bailyn, ApJ 544, 993-1015 (2000).

26. G. J. Sobczak, J. E. McClintock, R. A. Remillard, W. Cui, A. M. Levine, E. H. Morgan, J. A. Orosz and C. D. Bailyn, ApJ 531, 537-545 (2000).

27. M. R. Goad, T. P. Roberts, J. N. Reeves and P. Uttley, MNRAS 365, 191-198 (2006).

28. T. P. Roberts, Ap\&SS 311, 203-212 (2007).

29. A. Socrates and S. W. Davis, ApJ 651, 1049-1058 (2006).

30. P. S. Callahan, $A \& A$ 59, 127-131 (1977).

31. B. Czerny and M. Elvis, ApJ 321, 305-320 (1987).

32. A. M. Beloborodov, MNRAS 297, 739-746 (1998).

33. T. Kawaguchi, ApJ 593, 69-84 (2003).

34. Y. V. Artemova, G. S. Bisnovatyi-Kogan, I. V. Igumenshchev and I. D. Novikov, ApJ 637, 968-977 (2006).

35. J. Frank, A. R. King and D. Raine, "Accretion Power in Astrophysics", Cambridge University Press, Cambridge, 2002.

36. M. A. Abramowicz, B. Czerny, J. P. Lasota and E. Szuszkiewicz, ApJ 332, 646-658 (1988).

37. K. Ohsuga, S. Mineshige and K. Watarai, ApJ 596, 429-436 (2003)

38. K. Ohsuga, M. Mori, T. Nakamoto and S. Mineshige, ApJ 628, 368-381 (2005).

39. K. Watarai, T. Mizuno and S. Mineshige, ApJ 549, L77-L80 (2001).

40. K. Ebisawa, P. Zycki, A. Kubota, T. Mizuno, K. Watarai, ApJ 597, $780-797$ (2003).

41. J. Poutanen, G. Lipunova, S. Fabrika, A. G. Butkevich and P. Abolmasov, MNRAS 377, 1187-1194 (2007).

42. A. R. King, MNRAS 385, L113-L115 (2008).

43. S. K. Chakrabarti and L. G. Titarchuk, ApJ 455, 623-639 (1995).

44. L. G. Titarchuk, I. Lapidus and A. Muslimov, ApJ 455, 315-328 (1998).

45. S. Mandal and S. K. Chakrabarti, $A \& A$ 434, 839-848 (2005).

46. J. M. Miller, J. Homan and G. Miniutti, ApJ 652, L113-L116 (2006)

47. Z. Kuncic and G. V. Bicknell, ApJ 616, 669-687 (2004). 